\title{
Sleep quality and general health status of employees exposed to extremely low frequency magnetic fields in a petrochemical complex
}

\author{
Mohammad Reza Monazzam¹, Monireh Hosseini ${ }^{*}$, Laleh Farhang Matin³, Habib Allah Aghaei ${ }^{4}$,
} Hossein Khosroabadi ${ }^{5}$ and Ahmad Hesami ${ }^{5}$

\begin{abstract}
Background: Advances in science and technology of electrical equipment, despite increasing human welfare in everyday life, have increased the number of people exposed to Electro-Magnetic Fields (EMFs). Because of possible adverse effects on the health of exposed individuals, the EMFs have being the center of attention. This study was performed to determine possible correlation between Extremely Low Frequency Electro-Magnetic Fields (ELF EMFs) and sleep quality and public health of those working in substation units of a petrochemical complex in southern Iran.

Materials and method: To begin with, magnetic flux density was measured at different parts of a Control Building and two substations in accordance with IEEE std 644-1994. Subsequently, the questionnaires "Pittsburgh Sleep Quality Index" (PSQI) and "General Health Quality (GHQ)" were used to investigate relationship between ELF exposure level and sleep quality and public health, respectively. Both questionnaires were placed at disposal of a total number of 40 workers at the complex. The filled out questionnaires were analyzed by T-test, Duncan and the Chi-square tests.

Results: The obtained results revealed that $28 \%$ of those in case group suffered from poor health status and $61 \%$ were diagnosed with a sleep disorder.

However, all members in control group were in good health condition and only $4.5 \%$ of them had undesirable sleep quality.

Conclusion: In spite of a significant difference between the case and control groups in terms of sleep quality and general health, no significant relationship was found between the exposure level and sleep quality and general health. It is worth noting that the measured EMF values were lower than the standard limits recommended by American Conference of Industrial Hygienists (ACGIH). However, given the uncertainties about the pathogenic effects caused by exposure to ELF EMFs, further epidemiological studies and periodic testing of personnel working in high voltage substations are of utmost importance.
\end{abstract}

Keywords: Electromagnetic fields, ELF, Quality of sleep, General health

\section{Introduction}

Due to a substantial increase in the use of electrical energy in everyday life, number of people exposed to these fields in public or at workplace has increased dramatically [1-3].

Due to concerns about the possible harmful effects of exposure to EMFs, this physical agent has been the centre

\footnotetext{
* Correspondence: monirehosseini@ymail.com

${ }^{2}$ Department of Physics, Faculty of Basic Sciences, Islamic Azad

University- Tehran North Branch and Center for Air Pollution Research (CAPR), Institute for Environmental Research (IER), Tehran University of Medical Sciences, Tehran, Iran

Full list of author information is available at the end of the article
}

of attention in recent years [4,5]. Effects of EMFs on living organisms at different frequencies have long been under investigation by researchers worldwide. Kostoff and Lau in 2013 examined the scope of the accumulated effects on biological and health systems caused by simultaneous exposure to electromagnetic fields/radiation. They finally concluded although, there is a wide range of potential effects in which EMF plays a supportive or negative role; however, community consensus does not exist on these potential effects, either beneficial or adverse [6].

\section{Biomed Central}


Another research by Kroupová et al. in 2007 studied the effects of LFM on cytoskeleton and on the structure of chromatin in human cells. They could finally find no influence on higher order chromatin structure, however, certain changes on the level of cytoskeleton was detected by them [7].

A survey research was also done by Cârstea in 2012 on the impact of EMFs of the large power cables on human health. They could finally present an algorithm to stimulate the adverse health impacts [8]. What approved by all is harmfulness of overexposure to the EMFs. Anyhow, further researches seem necessary to study the effects of different exposure levels. Many studies have shown correlation between occupational and non-occupational exposure levels to EMFs and the risk of some types of cancer including leukemia, brain tumors and breast cancer. Such a relationship has not been confirmed by all.

On the contrary, the findings of dozens of studies revealed non-cancer effects of exposure to magnetic fields. A study by Chung-Yi et al. in 1996 showed that exposure to high EMFs can cause sleep disorders in women [9]. Bianchi and Thomas in 2013 also studied the current clinical standard for enumerating sleep physiology by EMF, from which basic sleep-wake stages are determined. It was found that the complexity of sleep physiology has inspired alternative metrics that are providing additional perceptions into the rich dynamics of sleep [10]. In another research by Hashish et al. in 2008, it was investigated the effects of continuous whole-body exposure to EMF on liver and blood parameters in mice. Their findings revealed relation between the ELF-EMF exposure and the oxidative stress through distressing redox balance which leads to physical disturbances [11].

Savitz et al. (1999) found a significant relationship between exposure to ELF EMFs and death from arrhythmia and Acute Myocardial Infarction (AMI) while no significant relationship they could find between ELF EMFs and is chemic heart disease [12]. In a survey by Sahl et al. (2002), no correlation was observed between AMI or chronic coronary heart disease and cumulative exposure to ELF-MF [13]. Noonan in 2002 performed a case-control study to investigate possible relationship between neurodegenerative diseases and occupational exposure to magnetic fields. They could finally find evidences of correlation between occupational exposure to MFs and Parkinson's disease. However, no relationship was reported by them on exposure to magnetic fields and Alzheimer's disease [14]. In a cohort study by Qiu et al. (2004) done in Stockholm, the risk of Alzheimer's disease and occupational exposure to magnetic fields was investigated. Their findings claimed that long-term occupational exposure to intense EMFs can increase the risk of dementia and Alzheimer's diseases among men while similar exposure conditions did not show any increase in the risk of Alzheimer's diseases in women [15]. Davanipour et al. (2007) found a positive correlation between exposure to EMF waves and Alzheimer's debases [16]. Seidler et al. in 2007 through a case-control study indicated that there is no significant correlation between exposure to magnetic fields and dementia, vascular dementia and Alzheimer's and diseases [17]. In a study by Yoausefi and Nasiri (2006) on employees of high voltage substations in Tehran, it was reported symptoms of depression, paranoia, obsessive-compulsive disorder, interpersonal sensitivity, anxiety, aggression, phobia or psychosis among employees [18]. Sharifi et al. (2009) showed that long-term exposure to magnetic fields, despite being below the limits recommended by the International Commission on Non-Ionizing Radiation Protection (ICNIRP), can cause exacerbation of intensify psychiatric disorders including sleep disorders [19].

Given the widespread and growing trend of releasing ELF EMFs and recent reports on their adverse effects on different body systems, this study was done to investigate possible correlation between ELF EMFs and the general health and sleep quality of employees working at two power substations in a petrochemical company in southern Iran.

\section{Material and methods}

In this study, in order to assess exposure level of personnel to high voltage substations, magnetic flux density was measured using grid method at different parts of the Control Building and two substations located in a petrochemical complex. Number of measurement points was 126 at Ethylene Substation, 24 at Boiler Substation and 70 at Control Room. Changing number of measurement points at each location is due to differences in dimension and shape of the rooms as well as layout of equipment and power panels. All measurements were done in duplicate at each grid point according to IEEE STD 644-1994 in normal site conditions [20]. The measurement instrument was a uniaxial HI-3604 ELF Survey Meter manufactured by Holaday Company. The device was held by the operator at the height of $1 \mathrm{~m}$ from the ground towards three axes $\mathrm{X}, \mathrm{Y}$ and $\mathrm{Z}$. The maximum value measured in three directions $\left(B_{\max }\right)$ was considered as the magnetic field strength at each point.

The device has the capability of measuring both 50/ $60 \mathrm{~Hz}$ - electric and magnetic fields from power transmission and distribution lines and equipment. It has a sensitivity of about $1 \mathrm{~V} / \mathrm{m}-199 \mathrm{KV} / \mathrm{m}$ against electric fields and $0.1 \mathrm{mG}-20 \mathrm{G}$ against magnetic fields [21]. The values of the magnetic flux density were compared with limits recommended by ACGIH [22]. Reviewing information contained in career records, the type of jobs and the numbers of people employed in each unit were specified. Table 1 presents records of job titles and characteristics of employees in each unit.

A total number of 40 people are working in a 12-hour irregular shift in the complex, all of whom are men. 
Table 1 Job title and characteristics of workers

\begin{tabular}{llll}
\hline Workplace & Job title & $\begin{array}{l}\text { Number of } \\
\text { people employed }\end{array}$ & Education \\
\hline $\begin{array}{llll}\text { Ethylene } \\
\text { substation }\end{array}$ & Engineer & 4 & Bachelor \\
& Technician & 4 & Associated diploma \\
& Worker & 1 & Diploma \\
Boiler substation & Engineer & 2 & Bachelor \\
& Site man & 3 & Associated diploma \\
& Operator & 4 & Diploma \\
& Operator & 16 & Bachelor \\
& Engineer & 6 & Bachelor \\
\hline
\end{tabular}

They are present in almost 10hours of their shift permanently in one place and leave their workplace just to use the restroom, eat, pray, etc. For a closer examination, the employees were divided in two groups of case and control. At each substation, there is more than one occupational group that all people, regardless of the different job titles, are in one room. The number of personnel working at the high voltage substations (Ethylene and Boiler) that are at risk of occupational exposure to magnetic fields was 18 people who were categorized in the case group. In other words, individuals in every substation, despite different job titles, are all in similar working conditions in terms of exposure to magnetic fields.

In the control group, those 22 people who are at the same working conditions and not exposed to occupational exposure to EMFs were categorized. Job titles in the control group include operators and engineers working respectively in the Control Room and Engineering Room. They were only exposed to background level of EMFs. In order to examine possible relationship between exposures to EMFs and sleep quality, the PSQI questionnaire was prepared while the General GHQ was used to detect correlation between exposure to EMFs and general health. The PSQI questionnaire was designed by Buysse et al. (1989) to help distinguish sleep disorders. It contains seven different questions consisting of individual terms of sleep quality, sleep duration, sleep latency, sleep efficiency, useful habits, sleep disturbances, use of sleeping pills and impaired daily function. Each question was given a score of 0 to 3 so that individuals' total score would range between 0 and 21 . According to the questionnaire designers, scores above 5 were considered as poor sleep quality [23].

The short form GHQ questionnaire including 28 questions was used to detect the general health effects of exposure to EMFs By which the despondences were asked about indisposition, discomfort and general health conditions with the main emphasis on their current psychological, physical and social issues. In other words, the questionnaire includes four categories of questions to diagnose somatic symptoms, anxiety and insomnia, social dysfunction and severe depression. The total score for each individual was obtained from the sum of the scores given to each category of the questions in these four fields of area [24]. Questions were scored by the Likert scale. In this research, a score of 23 was considered as the cut-off point of healthy and unhealthy status. According to which, scores higher than 23 was determined as unhealthy state. The above mentioned Standard questionnaires were filled out onsite. Participants were given adequate training before being asked to complete the questionnaires. The filled out questionnaires were then analyzed by MSTATC. The Chi-square, Duncan's and $t$-test were also used to detect possible relationship between the variables.

All participants provided informed written consent as approved by the ethics committee of Islamic Azad University, North-Tehran Branch.

\section{Results}

According to the obtained results, there was found no measurement point in the complex with the magnetic field amplitude higher than the standard limit of $1200 \mu \mathrm{T}$ recommended by ACGIH. The highest field strength was $49.90 \mu \mathrm{T}$ measured at the Boiler Unit at. The results from the Questionnaires PSQI and GHQ are listed in Table 2.

Data analysis by the $t$-test showed a significant difference between the case and control groups in terms of sleep quality at confidence level of $1 \%$. A significant difference was also found between the case group and the control group in terms of general health. The comparison results of these two groups in terms of sleep quality and general health are given in Tables 3 and 4 .

The results showed that $61 \%$ of workers in the case group suffered from poor sleep quality while $95.5 \%$ of those in control group had a good sleep quality and just $4.5 \%$ of them had poor sleep quality. The analysis results of the GHQ questionnaires showed that $72 \%$ of workers in the case group and all participants of the control group had a good condition of general health. The significant difference between the variance of the variables made it possible to compare the average of results obtained from the questionnaires filled out by each group members.

The Duncan Method was used to compare the mean values. Table 5 shows the outputs from the Duncan Method.

The PSQI analysis results by Duncan's Method revealed only three types of occupational groups including "workers" of the Ethylene Unit, "operators" of the Control Room and "engineers" of the "Engineering Room" enjoyed were categorized into the lowest class of "d" (Table 5) and enjoyed good quality sleep. Compared to other occupational groups, engineers at the Ethylene Unit had the worst sleep conditions and classified in the highest Dunkan class of " $a$ ". The sleep quality of those working in charge of engineers, site man and operator at Boiler Unit was poor, as well. 
Table 2 The results of the questionnaires by the control and case groups

\begin{tabular}{|c|c|c|c|c|c|}
\hline & Place & Job title & PSQI & GHQ & $B_{\max }(\mu \mathrm{T})$ \\
\hline \multirow[t]{2}{*}{ Control group } & Control room & Operator & 2.87 & 9.5 & 0.14 \\
\hline & Engineering room & Engineers & 1.83 & 5.5 & 0.05 \\
\hline \multirow[t]{6}{*}{ Case group } & Ethylene substation & Engineer & 14.33 & 31.5 & 40.43 \\
\hline & & Technician & 4.75 & 16.75 & \\
\hline & & Worker & 4 & 7 & \\
\hline & Boiler substation & Engineer & 12.5 & 20 & 49.90 \\
\hline & & Site man & 9 & 23 & \\
\hline & & Site operator & 5.5 & 12.25 & \\
\hline
\end{tabular}

Accordingly, the occupational group of engineers at Boiler Substation was classified between the classes " $a$ " and " $b$ " symbolized as $a b$ class. However, the occupational groups "site man" and "operators" of the unit were categorized in lower classes at a confidence level of $1 \%$.

Although, the mean value calculated for sleep quality of technicians at Ethylene Unit was lower than the index value however, they were given a score similar to those working at Boiler Unit so as all were classified at the same class. Both numbers, with a very little difference, are close to the index number of 5 , it can be concluded that these people are prone to crisis.

The analysis results of GHQ questionnaires showed that engineers working at Ethylene Unit were categorized in the highest class while those engineers working at Engineering Room were classified in the lowest class.

Only scores obtained for engineers at Ethylene Unit and site men of the Boiler Unit were higher than the index number of 23. This indicates personnel in these groups do not enjoy good health conditions. However, those in other occupational groups either in case group or particularly in the control group were given scores lower than the index number that indicates they are in a good conditions of health. The analysis results of the Chi-square Test for the PSQI questionnaires showed that the score of " $F$ " was significant at the confidence level of 1\% for operators at the Boiler Unit and Control Room as well as engineers at Engineering Room and technicians of the Ethylene Unit. In each occupational group, data distribution is at an acceptable level and sleep quality of the individuals does not differ much.

Table 3 Comparison of the control and case groups in terms of sleep quality and general health

\begin{tabular}{lllll}
\hline & Group & Mean & Variance & Standard deviation \\
\hline Sleep quality & Case & 9.25 & 20.51 & 4.53 \\
& Control & 2.63 & 1.13 & 1.06 \\
General health & Case & 7.50 & 11.14 & 3.34 \\
& Control & 20.70 & 77.17 & 8.78 \\
\hline
\end{tabular}

As the results suggest, the "F" score was not significant at confidence level of 1 for Engineers at Ethylene Unit operators and site men at Boiler Unit. Consequently, the results obtained for sleep quality of every individual differ and the data distribution is high. The "F" score of the GHQ questioners was found insignificant for all job titles at confidence level of $1 \%$. This revealed different general health condition of the employees where as data distribution is so high.

\section{Discussion}

As mentioned earlier, there was found no measurement point at which magnetic field amplitude value exceeds limits recommended by ACGIH. It should be noted that the maximum EMF measured in this research was 49.90 $\mu \mathrm{T}$. In a similar study by Sharifi Fard et al. (2011), there was also found no evidence of impermissible EMF at any point measured; the highest recorded values were 0.69 $\mu \mathrm{T}$ and $9.15 \mu \mathrm{T}$ recorded among the Control Rooms and near the switchgear, respectively [25]. Their findings showed no significant difference between the case and control groups in terms of psychological disorders [19].

Another investigation on the effect of extremely low frequency electromagnetic fields exposure on sleep quality in high voltage substations in city of Kerman and the suburbs shows that although there was a higher percentage of poor sleep quality on the case group than the control group, no statistically significant difference was perceived [26].

The results of the present study also show that compared with those in control group, the individuals who

Table 4 Results of the overall evaluation of data by $t$-test

\begin{tabular}{|c|c|c|}
\hline & Sleep quality & General health \\
\hline $\begin{array}{l}\text { Variance of the difference between } \\
\text { the means }\end{array}$ & 1.1663 & 10.3937 \\
\hline Standard deviation of the differences & 1.0799 & 3.2239 \\
\hline$t^{\prime}$ value & -6.1346 & -4.0944 \\
\hline Probability of $t^{\prime}$ & 0.0000 & 0.0004 \\
\hline
\end{tabular}


Table 5 Results of Duncan method

\begin{tabular}{|c|c|c|c|c|c|}
\hline Job title & Mean of quality of sleep & Class & Job title & Mean of general health & Class \\
\hline Engineers of Ethylene Unit & 14.25 & a & Engineers of Ethylene Unit & 31.50 & a \\
\hline Engineers of Boiler Unit & 12.75 & $a b$ & Site man of Boiler Unit & 23.00 & $b$ \\
\hline Site man of Boiler Unit & 9.00 & $b c$ & Engineers of Boiler Unit & 20.00 & $b c$ \\
\hline Operator of Boiler Unit & 5.50 & $\mathrm{~cd}$ & Technician of Ethylene Unit & 16.75 & c \\
\hline Technician of Ethylene Unit & 4.75 & $\mathrm{~cd}$ & Operator of Boiler Unit & 12.25 & $d$ \\
\hline Worker of Ethylene Unit & 4.00 & $d$ & Operator of Control Room & 9.50 & de \\
\hline Operator of Control Room & 3.00 & $d$ & Worker of Ethylene Unit & 7.00 & e \\
\hline Engineers of Engineering Room & 2.25 & $d$ & Engineers of Engineering Room & 5.50 & e \\
\hline
\end{tabular}

have been in occupational exposure to magnetic fields (the case group), may have poorer sleep quality and worse health status. However, no significant correlation was observed between exposure level and sleep quality and general health condition of the personnel $(\mathrm{p}<0.01)$. Yousefi and Nassiri in 2006 reported that symptoms of mental and behavioral disorders among people exposed to EMFs were significantly higher than those in control group. However, they could find no significant correlation between the above-mentioned symptoms and field strength and duration of exposure [18]. A case-control study by Wijngaarden et al. on exposure to EMFs showed that suicide rate was higher among those exposed to EMFs which could be due to the prevalence of depression among this group [27]. In a study by Zahiroddin et al. it was demonstrated that $17 \%$ of the control group and $32.7 \%$ of the case group were suspected of failing health $(\mathrm{p}<0.01)$ and it was indicated that incidence of mental health disorders have no relationship with variables such as marital status, age, work experience and shift work [28]. Gamberala et al. found symptoms of depression, paranoid, obsessivecompulsive disorder, sensitivity in individual and social behaviors, anxiety and aggression among workers exposed to EMFs [29]. Another study by Pearce et al. showed psychological side effects such as suicide, depression and uncontrollable emotional state in people living near power transmission lines and high voltage substations [30]. The findings of the present study on insignificant correlation of exposure level and sleep disorder and general health may be due to the small sample size of the study groups. Accordingly, further studies on larger populations are required. As mentioned earlier, the case group in this study included personnel of Ethylene and Boiler Units. In each unit, there were three job titles with similar working conditions and almost the same exposure level to magnetic fields. In spite of the similarity in working conditions and exposure level at each unit, the sleep quality and health status of each occupational group differ from each other $(\mathrm{p}<0.01)$. This is probably due to differences in physical and mental characteristics of the personnel. Employees with higher education degrees scored higher for sleep quality and general health status, showing that they had more undesirable quality of sleep and health condition than other staff. Zamanian et al. also revealed the psychological health level of people exposed to ELFEMFs was lower than that of control group. They could also find a significant correlation between deep depression and education level [31]. Despite numerous studies on the effects of EMFs on human, scientists have still failed to reach a consensus on the real side effects. Given the complexities of biological, psychological and social factors affecting human health, detailed examination on the effects of EMFs requires further researches at a larger scale.

\section{Conclusion}

The present study was done to investigate possible correlation between ELF EMFs and the general health and sleep quality of employees working at two power substations in a petrochemical company in southern Iran. As the findings suggest, in spite of a significant difference between the case and control groups in terms of sleep quality and general health, no significant relationship was found between the exposure level and sleep quality and general health. Although the amplitude of the magnetic fields was evaluated lower than the standard limits at all measurement points, however, more comprehensive studies should be done to detect possible side effects may cause by exposure to even below-standard EMFs. It is recommended to reduce exposure to the EMFs as much as possible through strategies such as increasing distance from EMF sources by remote control devices, training exposed workers and planning to diminish workplace stress.

\section{Competing interests}

The authors hereby declare that they have no competition of interests to be told

\section{Authors' contributions}

MRM: conception and design, analysis and interpretation, administrative, technical, or material support, supervision. $\mathrm{MH}$ : conception and design, data collection,analysis and interpretation, statistical expertise, writing the manuscript, critical revision of the manuscript. LFL: conception and design, administrative, technical, or material support.HAA: writing the manuscript. $\mathrm{HKH}$ : administrative, technical, or material support. $\mathrm{AH}$ : data collection, 
technical, or material support. All authors read and approved the final manuscript.

\section{Acknowledgments}

This study has been done with the support and funding of Iranian National Petrochemical Company (NPC). Hereby we would like to thank Dr. Nassiri, Director of HSE Department and other staff without whom this research would not be possible. Special thanks to all managers and employees at the target complex where the present study was carried out. We would also like to express our sincere gratitude for the services rendered by Ravian Danesh Mohit Company (Ravian D.M.) in providing insightful comments and proofreading the manuscript.

\section{Author details}

'Department of Occupational Hygiene, School of Public Health and Center for Air Pollution Research (CAPR), Institute for Environmental Research (IER), Tehran University of Medical Sciences, Tehran, Iran. ${ }^{2}$ Department of Physics, Faculty of Basic Sciences, Islamic Azad University- Tehran North Branch and Center for Air Pollution Research (CAPR), Institute for Environmental Research (IER), Tehran University of Medical Sciences, Tehran, Iran. ${ }^{3}$ Department of Physics, Faculty of basic sciences, Islamic Azad University- Tehran north branch, Tehran, Iran. ${ }^{4}$ Department of Occupational Health, School of Public Health, Tehran University of Medical Sciences, Tehran, Iran. ${ }^{5}$ National

Petrochemical Company, Tehran, Iran.

Received: 21 July 2013 Accepted: 21 April 2014

Published: 29 April 2014

\section{References}

1. Zhao CX, Huang K, Deng SZ, Xu NS, Chen J: Investigation of the effects of atomic oxygen exposure on the electrical and field emission properties of ZnO nanowires. App/ Surf Sci 2013, 270:82-89.

2. Marino C, Galloni P: Microwaves: Exposure and Potential Health Consequences. Encyclopedia of Environmental Health. Burlington, Book; 2011:765-773.

3. Negm A, Lorbergs A, Maclntyre NJ: Efficacy of low frequency pulsed subsensory threshold electrical stimulation vs placebo on pain and physical function in people with knee osteoarthritis: systematic review with meta analysis. Ostearthritis Cartilage 2013, 21(9):1281-1289.

4. Hardell L, Sage C: Biological effect from electromagnetic field exposure and public exposure standards. Biomed Pharmacother 2008, 62(2):104-109.

5. Frei $P$, Mohler E, Braun-Fahrländer C, Fröhlich J, Neubauer G, Röösli M: Cohort study on the effect of everyday life radio frequency electromagnetic field exposure on non-specific symptoms and tinnitus. Environ Int 2012, 38(1):29-36.

6. Kostoff RN, Lau CGY: Combined biological and health effects of electromagnetic fields and other agents in the published literature. Technol Forecast Soc 2013, 80(7):1331-1349.

7. Kroupová J, Bártová E, Fojt L, Strašák L, Kozubek S, Vetterl V: Low-frequency magnetic field effect on cytoskeleton and chromatin. Bioelectrochemistry 2007, 70(1):96-100.

8. Cârstea D: Electromagnetic field of the large power cables and impact on the human health. J WSEAS Trans Circuits Syst 2012, 11(1):1-10.

9. Chung-Yi L, Sung FC, Lin RS: Residential exposure to power frequency magnetic field and sleep disorders among women in an urban community of northern Taiwan. Sleep 2002, 25(4):428-432.

10. Bianchi MT, Thomas RJ: Technical advances in the characterization of the complexity of sleep and sleep disorders. Prog Neuropsychopharmacol Biol Psychiatry 2013, 45(1):277-286.

11. Hashish AH, El-Missiry MA, Abdelkader HI, Abou-Saleh RH: Assessment of biological changes of continuous whole body exposure to static magnetic field and extremely low frequency electromagnetic fields in mice. Ecotoxicol Environ Saf 2008, 71(3):895-902.

12. Savitz DA, Liao D, Sastre A, Kleckner RC, Kavet R: Magnetic field exposure and cardiovascular disease mortality among electric utility workers. Am J Epidemiol 1999, 149(2):135-142.

13. Sahl J, Mezei G, Kavet R, McMillan A, Silvers A, Sastre A, Kheifets L: Occupational magnetic field exposure and cardiovascular mortality in a cohort of electric utility workers. Am J Epidemiol 2002, 156(10):913-918.

14. Noonan CW, Reif JS, Yost M, Touchstone J: Occupational exposure to magnetic fields in case-referent studies of neurodegenerative diseases. Scand J Work Environ Health 2002, 28(1):42-48.
15. Qiu C, Fratiglioni L, Karp A, Winblad B, Bellander T: Occupational exposure to electromagnetic fields and risk of Alzheimer's disease. Epidemiology 2004, 15(6):687-694.

16. Davanipour Z, Tseng CC, Lee PJ, Sobel E: A case control study of occupational magnetic field exposure and Alzheimer's disease: results from the California Alzheimer's Disease Diagnosis and Treatment Centers. BMC Neurol 2007, 7:13.

17. Seidler A, Geller P, Nienhaus A, Bernhardt T, Ruppe I, Eggert S, Hietanen M, Kauppinen T, Frölich L: Occupational exposure to low frequency magnetic fields and dementia: a case-control study. Occup Environ Med 2007, 64(2):108-114.

18. Yousefi HA, Nasiri P: Psychological effect of occupational exposure to electromagnetic fields. J Res Health Sci 2006, 6(1):18-21.

19. Sharifi M, Nasiri P, Monazzam MR: Measurement of the magnetic fields of high voltage substations ( $230 \mathrm{KV}$ ) in Tehran (Iran) and assessment of their effects. Iran J Med Phys 2010, 7(3):49-56.

20. Institute of Electrical \& Electronics Engineers (IEEE): IEEE Standard Procedures for Measurement of Power Frequency Electric and Magnetic Fields from AC Power lines. 1994. [Standard] IEEE.

21. Holaday Industries Inc: HI-3604 ELF survey meter user's manual. 2008. http:// manualbrand.com/hi-3604-elf-survey-meter-users-manual.htmlwebcite. Accessed June 30, 2012.

22. ACGIH: TLVS and BEIs Based on the Documentation of the Threshold Limit Values for Chemical Substances and Physical Agents and Biological Exposure Indices. Ohio, USA: American Conference of Governmental Industrial Hygienists; 2010:153-154.

23. Buysse DJ, Reynolds CF, Monk TH, Berman SR, Kupfer DJ: The Pittsburgh Sleep Quality Index: A new instrument for psychiatric practice and research. Psychiatry Res 1989, 28(2):193-213.

24. Goldberg DP, Hillier VF: A scale version of general health questionnaire. Psychol Med 1979, 9:131-145.

25. Sharifi Fard M, Nasiri P, Monazzam MR: Measurement of the magnetic fields of high voltage substations (230kV) in Tehran and comparison with the ACGIH threshold limit value. Radiat Pro Dosimetry 2011, 145(4):421-425.

26. Barsam T, Monazzam MR, Haghdoost AA, Ghotbi MR, Dehghan SF: Effect of extremely low frequency electromagnetic field exposure on sleep quality in high voltage substations. Iranian J Environ Health Sci Eng 2012, 9(1):15.

27. Wijngaarden EV, Savitz DA, Kleckner RC, Loomis D: Exposure to electromagnetic fields and suicide among electric utility workers. Occup Environ Med 2000, 57(4):258-263.

28. Zahiroddin AR, Shafiee AR, Mahdavi HN: Mental health status of employees in substations of electromagnetic fields at extremely low frequency in Tehran. Iranian J Environ Health Sci Eng 2006, 3(3):217-221.

29. Gamberala F: Physiological and psychological effects of exposure to extremely low frequency electric and magnetic fields on humans. Scand J Work Environ Health 1990, 1:51-54.

30. Beale IL, Pearce NE, Conroy DM, Henning MA, Murrell KA: Psychological effects of chronic exposure to $50 \mathrm{~Hz}$ magnetic fields in humans living near extra-high-voltage transmission lines. Bioelectromagnetics 1997, 18(8):584-594.

31. Zamanian Z, Gharepoor S, Dehghany M: Effects of Electromagnetic fields on mental health of the staff employed in gas power plants, Shiraz. Pakistan J Biol Sci 2010, 13(19):956-960.

\section{doi:10.1186/2052-336X-12-78}

Cite this article as: Monazzam et al:: Sleep quality and general health status of employees exposed to extremely low frequency magnetic fields in a petrochemical complex. Journal of Environmental Health Science \& Engineering 2014 12:78. 